\title{
Supporting Information: Two-dimensional Electronic Spectroscopy Using Rotating Optical Flats
}

\author{
Patrick C. Tapping, ${ }^{\dagger}$ Yin Song, ${ }^{\ddagger}$ Yoichi Kobayashi, ${ }^{\llbracket}$ Gregory D. Scholes,$\stackrel{\S}{ }$ and \\ Tak W. Kee*,† \\ $\dagger$ Department of Chemistry, The University of Adelaide, North Terrace, Adelaide, South \\ Australia 5005, Australia \\ $\ddagger$ Department of Physics, University of Michigan, 450 Church Street, Ann Arbor, Michigan \\ 48109, United States of America \\ -Department of Applied Chemistry, College of Life Sciences, Ritsumeikan University, 1-1-1 \\ Nojihigashi, Kusatsu, Shiga 525-8577, Japan \\ $\S$ Department of Chemistry, Princeton University, Washington Road, Princeton, New \\ Jersey 08540, United States of America \\ E-mail: tak.kee@adelaide.edu.au \\ Phone: +61-8-8313-5314
}




\section{Pulse Sequence and Characterization}

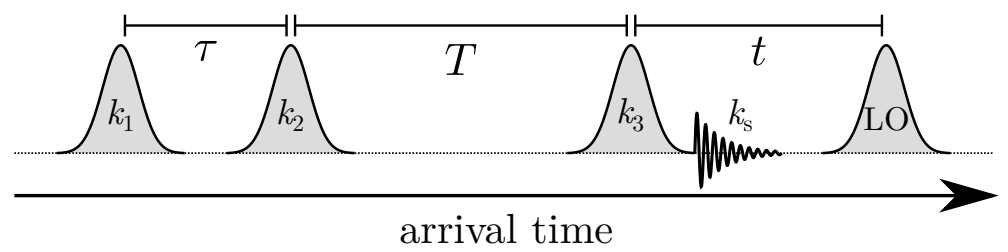

Figure S1: The pulse sequence for the 2D electronic spectroscopy experiments. The delay between the first two pulses, $\boldsymbol{k}_{1}$ and $\boldsymbol{k}_{2}$, defines the coherence time, $\tau$, while the delay between the second and third pulse, $\boldsymbol{k}_{3}$, is the population time, $T$. S1 The detected signal, $\boldsymbol{k}_{\mathrm{s}}$, is emitted following the third pulse and is detected interferometrically with an attenuated local oscillator (LO) pulse separated by some time, $t$. Note that in the depicted pulse sequence the LO arrives last, though it may instead be placed before the first excitation pulse. The choice is somewhat arbitrary, requiring the pulses to be close enough in time so that the produced interferogram can be resolved by the spectrograph and detector, but adequate temporal separation is also needed so that the signal peak can be readily extracted after the Fourier transform of the interferogram.
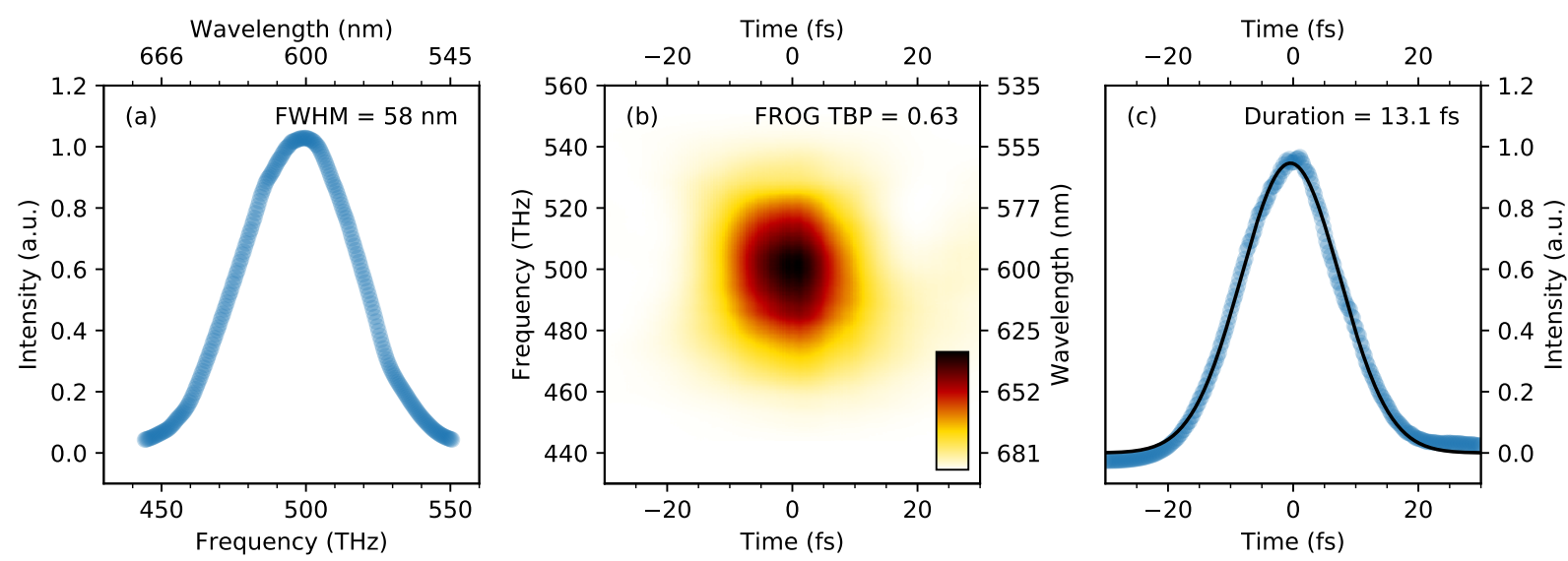

Figure S2: (a) The spectrum of the compressed laser pulse. (b) FROG image obtained from methanol in a TG-FROG configuration. (c) Analysis of the FROG signal over all frequencies (blue), and using a Gaussian function (black) to show a pulse duration of $13.1 \mathrm{fs}$. The time-bandwidth product (TBP) for a Gaussian pulse is $\sim 0.44$ at the transform limit. 


\section{Spectrometer Stability}

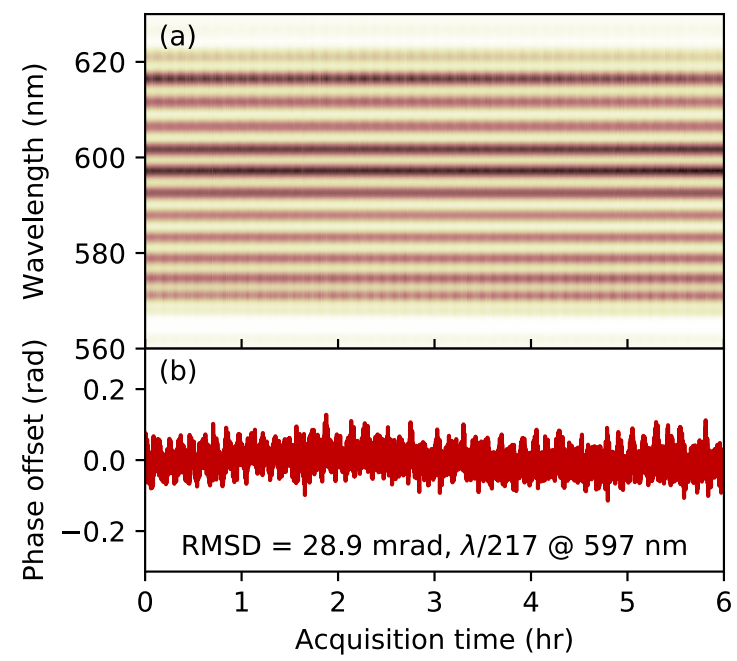

Figure S3: (a) Interference pattern and (b) computed phase stability of the spectrometer over a long time scale. While the short time scale data shown in the main text approximates the duration of a single $2 \mathrm{D}$ scan (for a given population time, $T$ ), the long time scale is indicative of stability over the several hours required to collect a complete data set. Sample was a cresyl violet solution with delays fixed so that $\tau=0 \mathrm{fs}, T=195 \mathrm{fs}$ and spectra acquired at $2 \mathrm{~s}$ intervals. The oscillatory nature of the phase offset is related to air temperature fluctuations associated with the laboratory air-conditioning. 


\section{Phasing via Projection Slice Theorem}

The phasing is performed by multiplying the rephasing and nonrephasing parts of the data by $\exp \left(2 \pi i f_{3}(t+\Delta t)+i \phi\right)$, where $\phi=\phi_{\mathrm{R}}$ or $-\phi_{\mathrm{NR}}$ ranges from 0 to $2 \pi$ and is a constant phase offset parameter for the rephasing or nonrephasing parts which compensates for small errors in the $\boldsymbol{k}_{1}$ and $\boldsymbol{k}_{2}$ pulse timings. The $\Delta t$ parameter represents the error in the measurement of the signal and LO separation time, $t$, used to correct the linear spectral phase. The value of $t$ is typically measured interferometrically in a separate experiment using the nonresonant response from methanol with $\tau=T=0 .{ }^{\text {S2 }}$ However, if the solvent response from a sample is large, the recorded spectrum from the data set at $\tau=T=0$ may also be used to estimate $t$ without performing an independent measurement. Determination of $\phi_{\mathrm{R}}, \phi_{\mathrm{NR}}$ and $\Delta t$ is by use of the projection slice theorem, ${ }^{\mathrm{S} 2}{ }^{\mathrm{S}} 4$ which states that the real parts of the sum of the rephasing and nonrephasing data, when collapsed along the excitation axis, should resemble data collected in a pump-probe-type experiment. For the apparatus shown in Figure 1b of the main text, the pump-probe data can be collected by blocking $\boldsymbol{k}_{1}$ and $\boldsymbol{k}_{3}$, using $\boldsymbol{k}_{2}$ as the pump and the LO as the probe. A global fitting algorithm is used to find the parameters that minimize the differences between the pump-probe and $2 \mathrm{D}$ projection data matrices. Previous work has shown phasing using three parameters (two linear, one constant) gives superior results to two (constant) parameter methods. ${ }^{\mathrm{S} 2, \mathrm{~S} 5}$ In this work, we use also use three parameters, but instead use one linear and two constant. Given that the linear phase is determined by $t$, the time between the signal and LO, and that the timings of $\boldsymbol{k}_{3}$ and the LO remain fixed, this choice of parameters may better physically represent the unknowns in the phase. Identical results were obtained when phasing using two linear and one constant parameter, or with the inclusion of an additional quadratic phase term. 

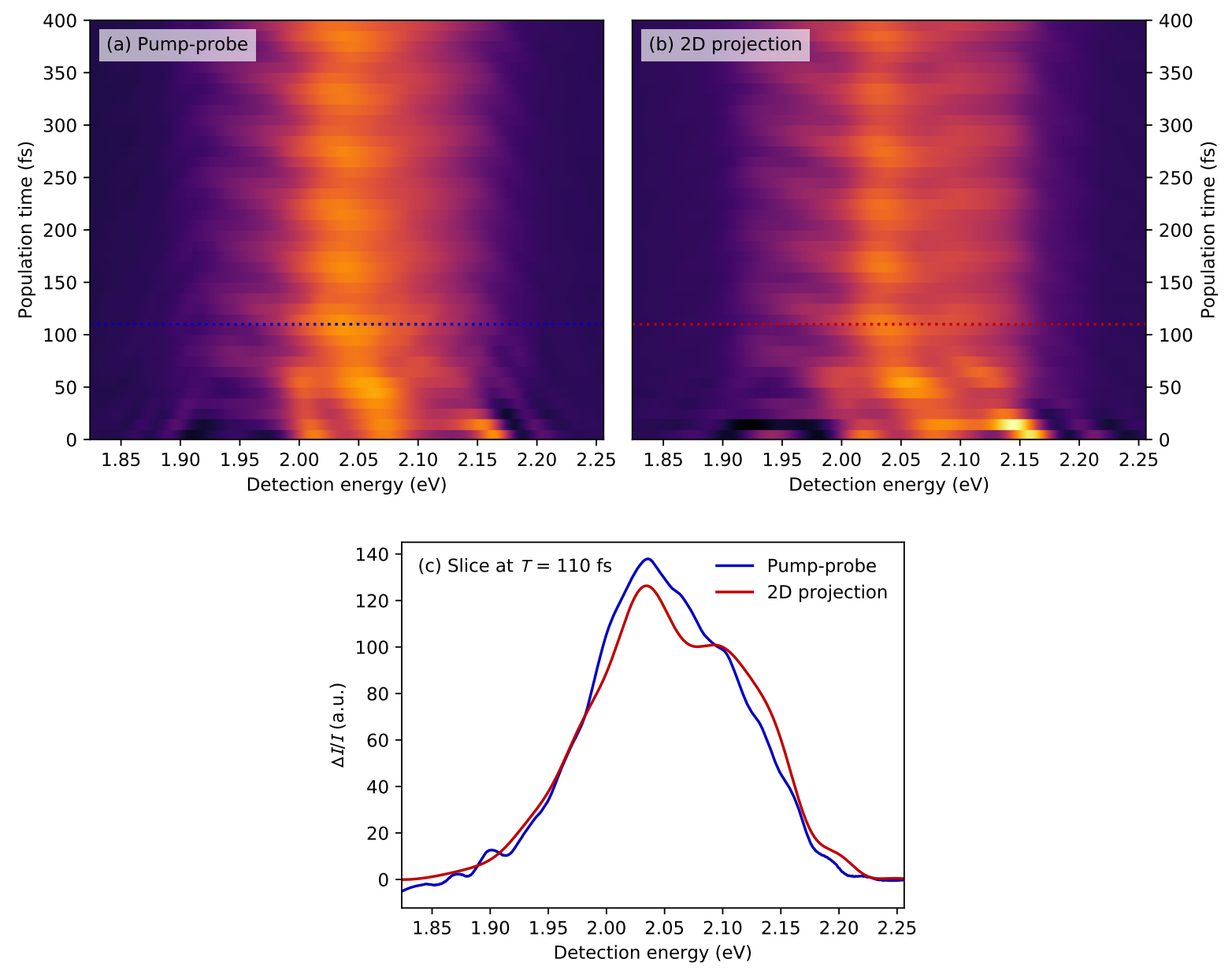

Figure S4: Comparison of the cresyl violet (a) pump-probe experiment versus (b) the 2D projection slice after fitting of phase parameters. A slice though a population time of $T=$ $110 \mathrm{fs}$ is indicated by the dotted lines and shown in (c). Note that the global fitting algorithm uses the complete data matrices, with the scale and time-zero offset of the pump-probe data being additional fitting parameters. 


\section{Cresyl Violet Data}

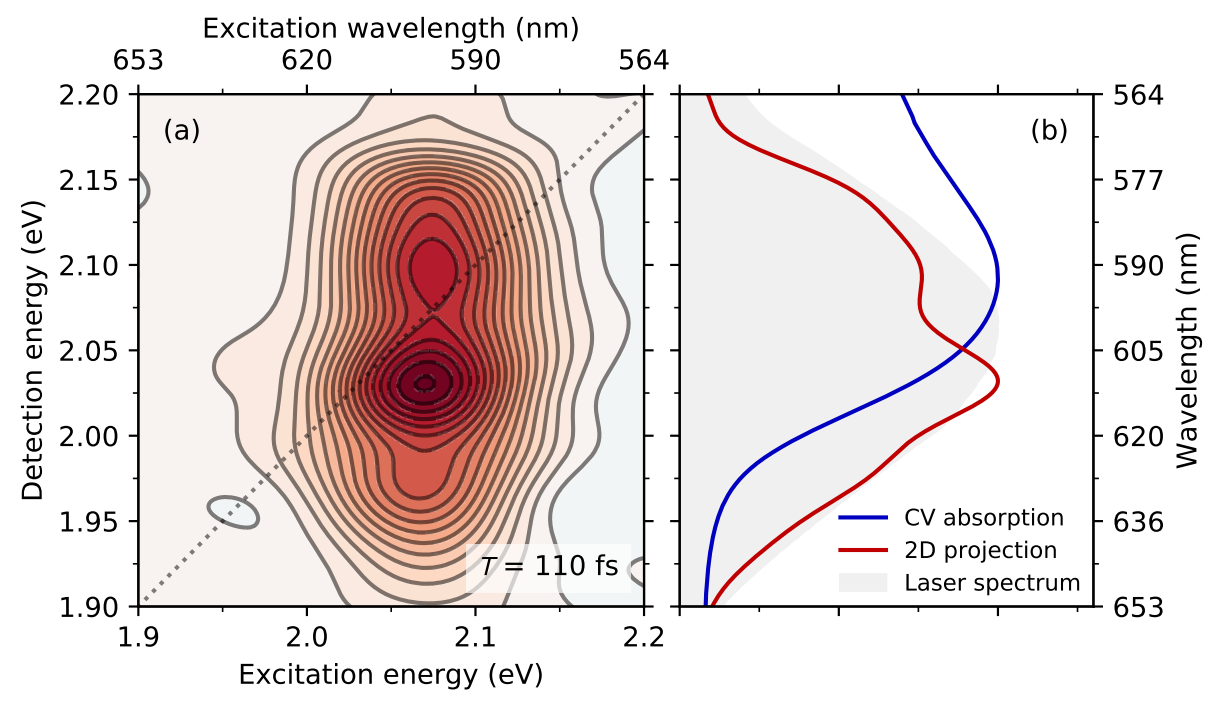

Figure S5: The absorptive 2D spectra of cresyl violet at a population time of $T=110 \mathrm{fs}$ is shown in (a), and the projection onto the detection axis (red curve) is compared to the absorption spectrum of cresyl violet (blue curve) in (b). The spectrum of the laser pulse is indicated with the grey shaded area. The $2 \mathrm{D}$ spectrum is composed of the ground-state bleach and stimulated emission signals, but note that detection is only possible where there is sufficient laser spectral intensity. 

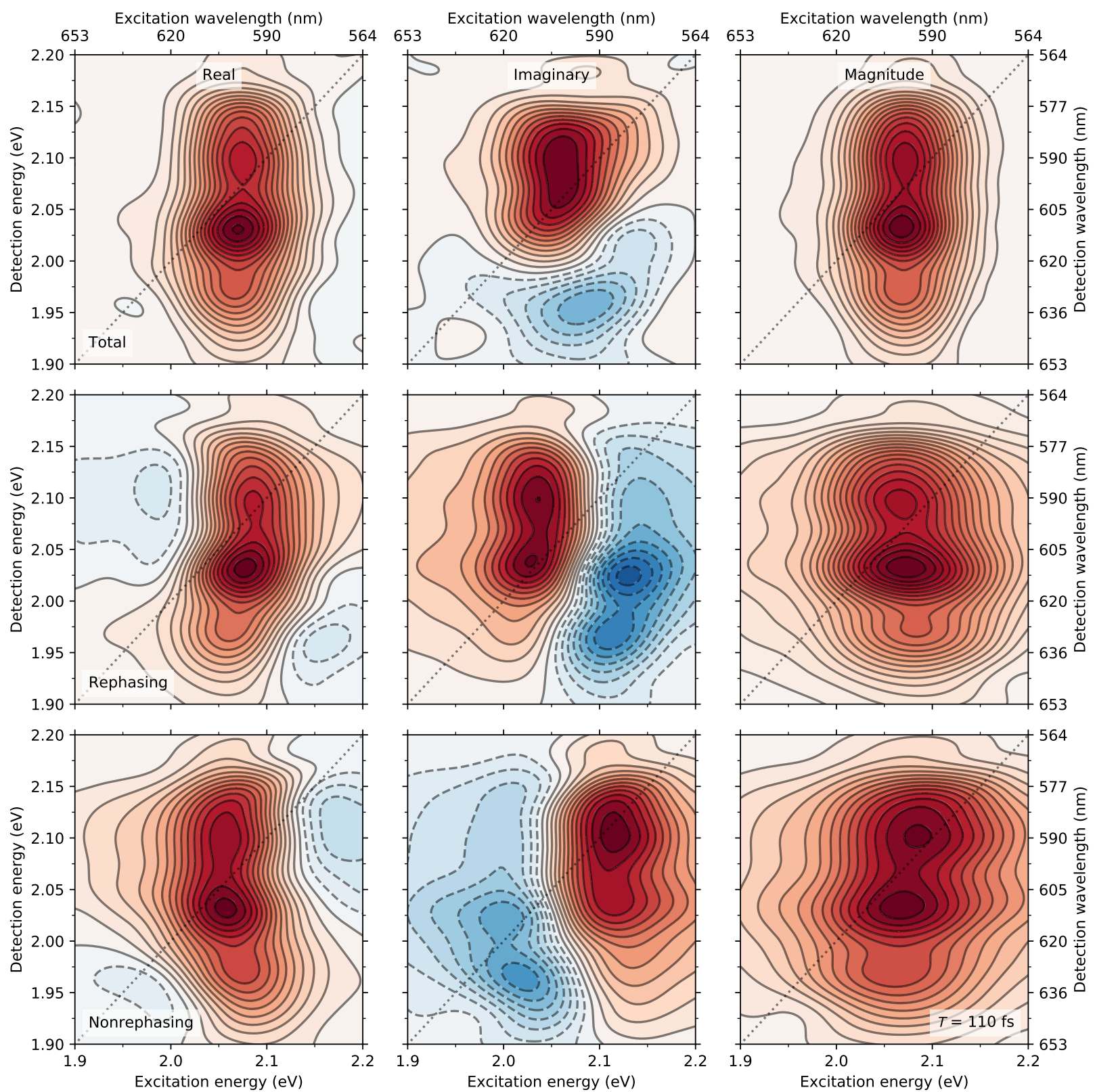

Figure S6: Complete view of a 2D data slice of cresyl violet at a population time of $T=110 \mathrm{fs}$ showing real, imaginary and magnitude of the total as well as separated rephasing and nonrephasing components. 


\section{Rotational Delay Stage Model}

The rotational delay stages comprise a pair of optical flats, with the second optic required to compensate for the beam deviation. Considering just a single piece of glass, the total delay due to the angle of the glass is the sum of contributions from increased path through glass, reduced path through air on the outer side of the glass pair, and increased path length through air on the inner side:

$$
\Delta t=\Delta t_{\mathrm{g}}+\Delta t_{\mathrm{out}}+\Delta t_{\mathrm{in}}
$$
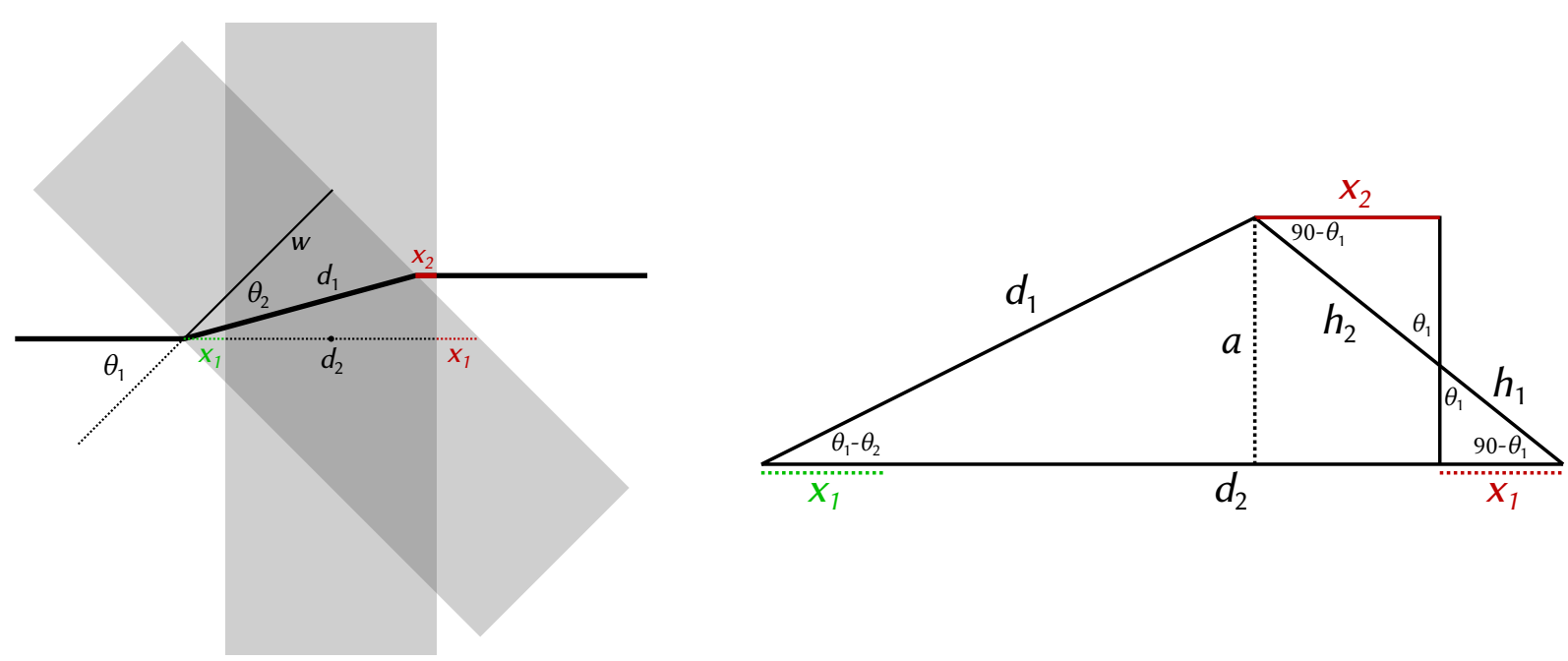

Figure S7: Diagram indicating increase in glass path and decrease in air path as glass is rotated resulting in delay of a transmitted laser pulse.

The incident angle of the laser beam relative to the normal of the glass is $\theta_{1}$. By Snell's law, refraction angle depends on the refractive indices, $n_{1}$ and $n_{2}$, of the media (air and glass) so the internal angle is

$$
\theta_{2}=\arcsin \left(n_{1} \sin \left(\theta_{1}\right) / n_{2}\right)
$$

The new path length through the glass, $d_{1}$, is dependent on the internal angle and the thickness of the glass, $w$, by

$$
\begin{aligned}
d_{1} & =w / \cos \theta_{2} \\
& =w / \cos \left(\arcsin \left(n_{1} \sin \left(\theta_{1}\right) / n_{2}\right)\right) .
\end{aligned}
$$

The change in glass path length is $\Delta d_{\mathrm{g}}=d_{1}-w$, thus the delay introduced due to additional glass is

$$
\begin{aligned}
\Delta t_{\mathrm{g}} & =\Delta d_{\mathrm{g}} n_{2} / c \\
& =n_{2}\left(w / \cos \left(\arcsin \left(\left(n_{1} / n_{2}\right) \sin \left(\theta_{1}\right)\right)\right)-w\right) / c,
\end{aligned}
$$

where $c$ is the speed of light. 
The difference in path length through air on an outer side of the glass pair is

$$
\Delta d_{\text {out }}=x_{1}=\left(d_{2}-w\right) / 2,
$$

so the delay introduced is

$$
\begin{aligned}
\Delta t_{\text {out }} & =-\frac{n_{1}}{2 c}\left(d_{2}-w\right) \\
& =-\frac{n_{1}}{2 c}\left(w / \cos \left(\theta_{1}\right)-w\right) .
\end{aligned}
$$

By similar triangles, the difference in path length through air on an inner side of the glass pair is

$$
\Delta d_{\text {in }}=x_{2}=h_{2} x_{1} / h_{1} .
$$

The hypotenuse lengths $h_{1}=x_{1} / \sin \theta$ and $h_{2}$ can also be found using similar triangles:

$$
a=d_{1} \sin \left(\theta_{1}-\theta_{2}\right)
$$

and

$$
a=\left(h_{1}+h_{2}\right) \sin \left(90-\theta_{1}\right)
$$

so

$$
\begin{aligned}
d_{1} \sin \left(\theta_{1}-\theta_{2}\right) & =\left(h_{1}+h_{2}\right) \sin \left(90-\theta_{1}\right) \\
h_{1}+h_{2} & =d_{1} \sin \left(\theta_{1}-\theta_{2}\right) / \sin \left(90-\theta_{1}\right) \\
& =d_{1} \sin \left(\theta_{1}-\theta_{2}\right) / \cos \left(\theta_{1}\right) \\
h_{2} & =d_{1} \sin \left(\theta_{1}-\theta_{2}\right) / \cos \left(\theta_{1}\right)-h_{1} \\
& =d_{1} \sin \left(\theta_{1}-\theta_{2}\right) / \cos \left(\theta_{1}\right)-x_{1} / \sin \theta .
\end{aligned}
$$

Substituting into eq 7 and simplifying gives

$$
\begin{aligned}
\Delta d_{\text {in }}=x_{2} & =h_{2} x_{1} / h_{1} \\
& =d_{1} \tan \left(\theta_{1}\right) \sin \left(\theta_{1}-\theta_{2}\right)+\left(w-d_{2}\right) / 2
\end{aligned}
$$

and thus the delay is

$$
\Delta t_{\text {in }}=\frac{n_{1}}{c}\left(d_{1} \tan \left(\theta_{1}\right) \sin \left(\theta_{1}-\theta_{2}\right)+\left(w-d_{2}\right) / 2\right)
$$

Note that this is an idealised model and thus does not account for real-world effects such as glass surface roughness. See the main text for discussion of calibration method required to achieve the accuracy required for the $2 \mathrm{D}$ spectroscopy experiments. 


\section{Comparison of Delay Components for Rotating Optical Flats versus Translating Wedges}
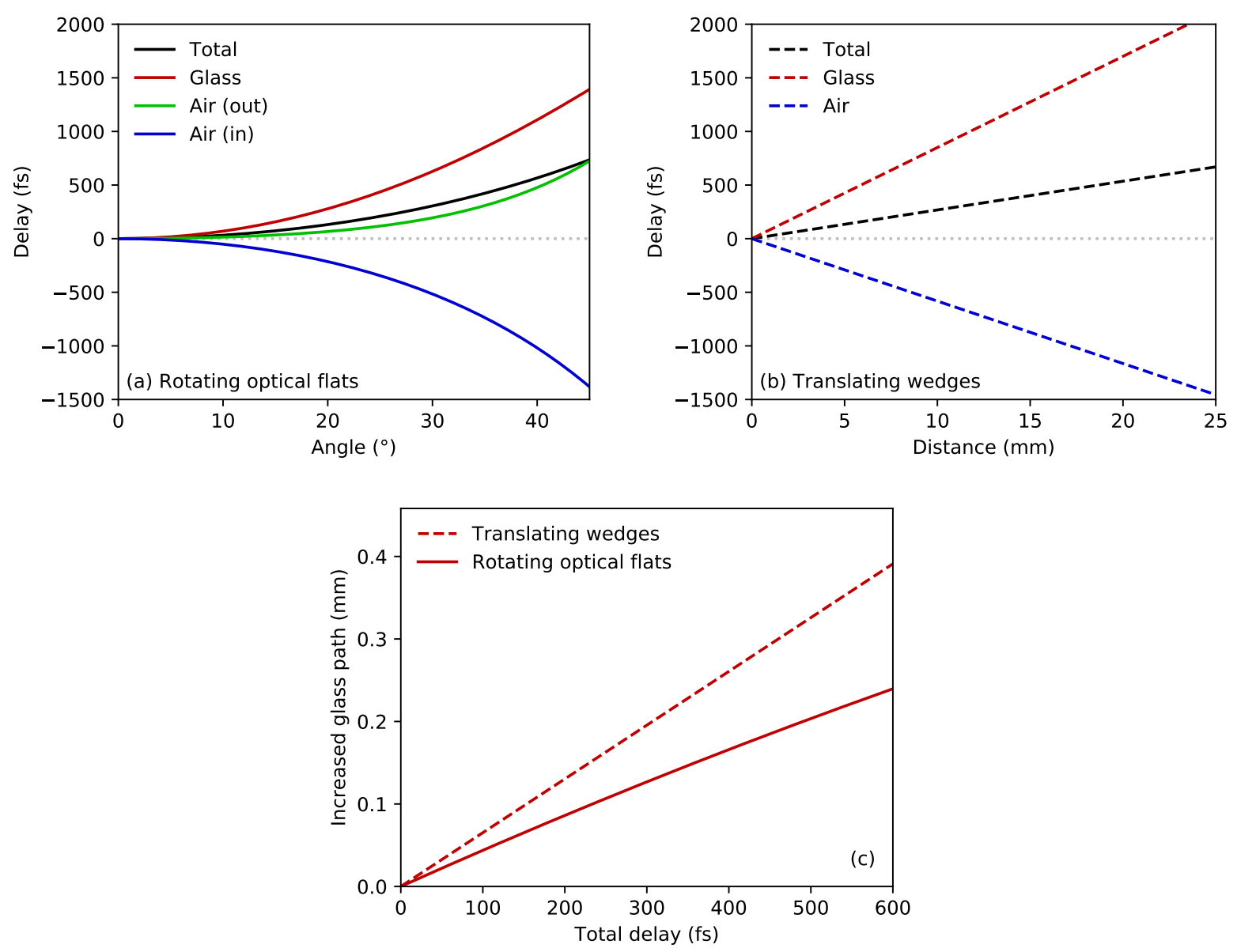

Figure S8: The total delay for both (a) the rotating optical flats and (b) translating wedges is dependent on the increase in effective glass thickness and accompanying change in path through air. In (a), the total delay (eq 1) as a function of glass rotation angle is shown, along with its glass and air contributions (eqs 4, 6 and 12). Note that because of the beam path deviation between the two optical flats, there is a component of increased path through air (green curve) which contributes positively to the total delay. In (b), the increased glass path of the translating wedge design is always accompanied by an equal decrease in air path, and the delay is entirely due to the different refractive indices of the two media. Panel (c) shows the increase in glass path length as a function of total programmed delay time (black curves in a and b) for rotating optical flats and translating wedges. The rotating optical flats require a shorter path through glass for the same total delay as translating wedges, which results in reduced spectral dispersion. Modeling is based on fused silica $(n=1.46)$, using a pair of $1 \mathrm{~mm}$ optical flats or wedges cut at $1^{\circ}$. Changing the thickness or cut angle changes the $\mathrm{y}$-axis scale, but not the curve shapes. 


\section{Laser Transmission versus Optical Flat Angle}

The transmission of the laser beams through the optical flats varies with angle according to the Fresnel equations. ${ }^{\text {S6 }}$ Transmission of a p-polarized beam through a single interface as a function of angle is given by

$$
T_{\mathrm{p}}\left(\theta, n_{1}, n_{2}\right)=1-\left|\frac{n_{1} \sqrt{1-\left(\frac{n_{1}}{n_{2}} \sin \theta\right)^{2}}-n_{2} \cos \theta}{n_{1} \sqrt{1-\left(\frac{n_{1}}{n_{2}} \sin \theta\right)^{2}}+n_{2} \cos \theta}\right|^{2},
$$

and for an s-polarized beam

$$
T_{\mathrm{s}}\left(\theta, n_{1}, n_{2}\right)=1-\left|\frac{n_{1} \cos \theta-n_{2} \sqrt{1-\left(\frac{n_{1}}{n_{2}} \sin \theta\right)^{2}}}{n_{1} \cos \theta+n_{2} \sqrt{1-\left(\frac{n_{1}}{n_{2}} \sin \theta\right)^{2}}}\right|^{2},
$$

where $n_{1}$ or $n_{2}$ are the refractive indices of the initial or final medium, and $\theta$ is the angle of incidence.

For the first (air-glass) interface of a fused silica optical flat, $n_{1}=n_{\text {air }} \approx 1, n_{2}=n_{\text {glass }}=$ 1.46 and $\theta$ is the rotation angle of the optical flat. For the second (glass-air) interface, Snell's law gives the the internal angle of incidence as

$$
\theta_{2}=\arcsin \left(\frac{n_{1}}{n_{2}} \sin \theta\right) .
$$

The transmission through a single optical flat is thus

$$
T_{2}(\theta)=T\left(\theta, n_{\text {air }}, n_{\text {glass }}\right) T\left(\theta_{2}, n_{\text {glass }}, n_{\text {air }}\right),
$$

and for a pair of optical flats

$$
T_{4}(\theta)=T_{2}(\theta)^{2}
$$

where $T$ is given by eq 13 or 14 for the appropriate polarization.

The intensity of the transmitted light as a function of optical flat rotation angle was measured for both the p-polarized $\boldsymbol{k}_{\mathbf{1}}$ and s-polarized $\boldsymbol{k}_{\mathbf{2}}$ beams (see Figure 1b of the main text) and found to match that predicted by eq 17, as shown in Figure S9. As the the pump-probe signal is linearly dependent on excitation intensity, eq 17 can be applied to the pump-probe data to correct for the variation in transmission with optical flat rotation.

In the 2D experiment, the third-order response is measured, thus the signal is dependent on the product of laser beam intensities. For this reason, the opposing effects of the $\mathrm{p}$ and s polarizations with optical flat rotation cause a minimal effect on the third-order signal intensity, with a $5 \%$ difference across the full delay range of $0-550$ fs (using a $\sim 40^{\circ}$ rotation), as shown as the black curve in Figure S9. 


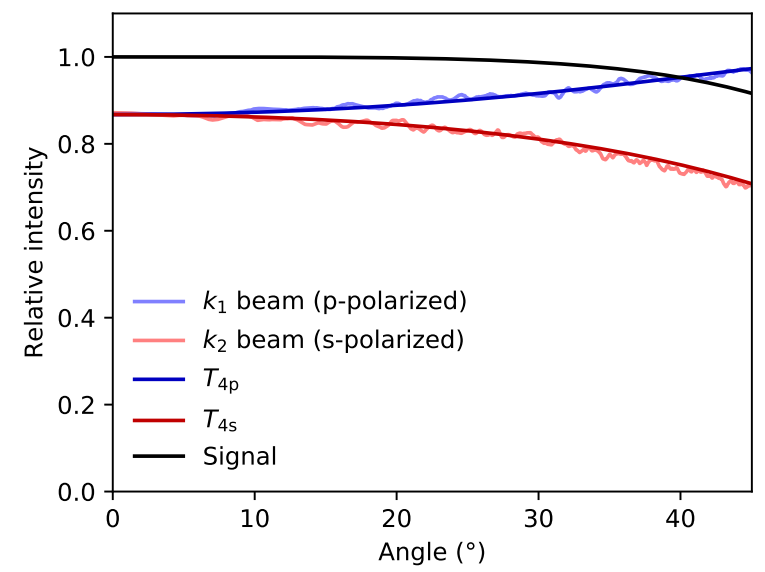

Figure S9: The measured laser beam transmission as a function of optical flat rotation angle for the p-polarized $\boldsymbol{k}_{1}$ and s-polarized $\boldsymbol{k}_{1}$ beams, with comparisons to theoretical transmission values given by eq 17 for p- or s-polarized light. The relative change in intensity of the thirdorder signal given by the product of the relative changes in laser beam intensities is indicated with the black curve. 


\section{Spectral Dispersion Measured via Interferometry}

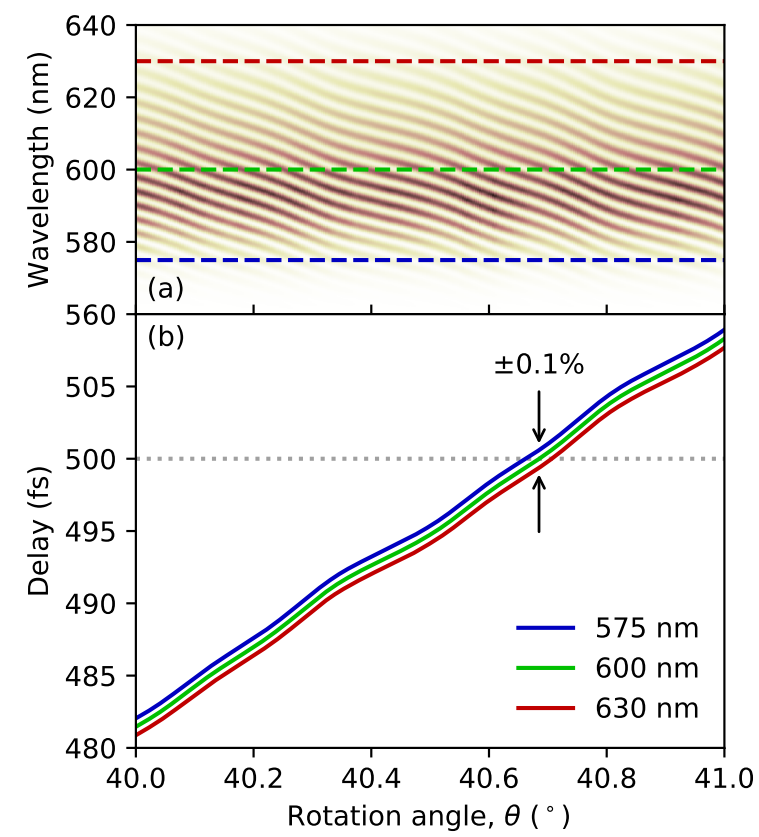

Figure S10: (a) Segment of a spectral interference pattern used to construct a delay-to-angle calibration curve. The analysis procedure gives a curve for each detection wavelength where there is sufficient intensity, allowing direct measurement of the spectral dispersion as the optical path length through glass is increased. (b) Measured optical delay as a function of rotation angle of the optical flats at three wavelengths corresponding to the blue edge, peak, and red edge of the laser spectrum, indicated by the dashed lines in (a). In this configuration, spectral dispersion due to the changing glass path results in a $\pm 0.1 \%$ error in delay time across the full rotation range. 


\section{Beam Displacement using Rotating Optical Flats versus Translating Wedges}

The beam displacement, $x$, after passing through wedges cut at angle, $\phi$, separated by distance, $d$ is

$$
x=\frac{d \tan \left(\arcsin \left(n_{2} \sin (\phi) / n_{1}\right)-\phi\right)}{1-\tan (\phi) \tan \left(\arcsin \left(n_{2} \sin (\phi) / n_{1}\right)-\phi\right)},
$$

where $n_{1}$ and $n_{2}$ are the refractive indices of air and the glass, respectively.

(a)

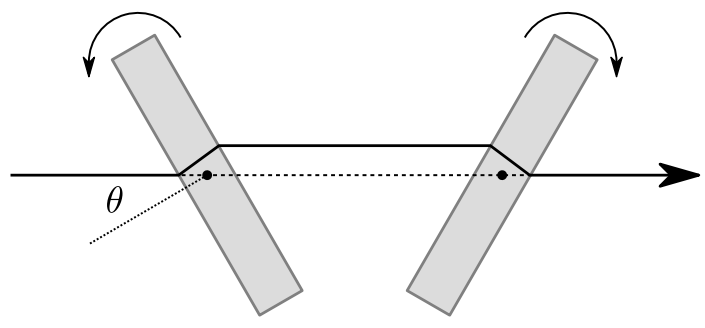

(b)

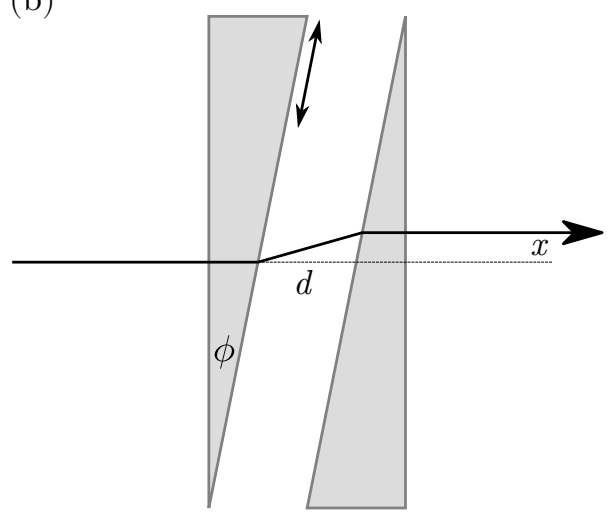

Figure S11: Using rotating optical flats (a), the beam displacement caused by passing through the first optic varies with rotation angle, $\theta$, but is compensated with the second optic, resulting in no net change in the beam position. With translating wedges (b), the wedge angle, $\phi$, and separation distance, $d$, must be kept small to minimize the final beam displacement, $x$.
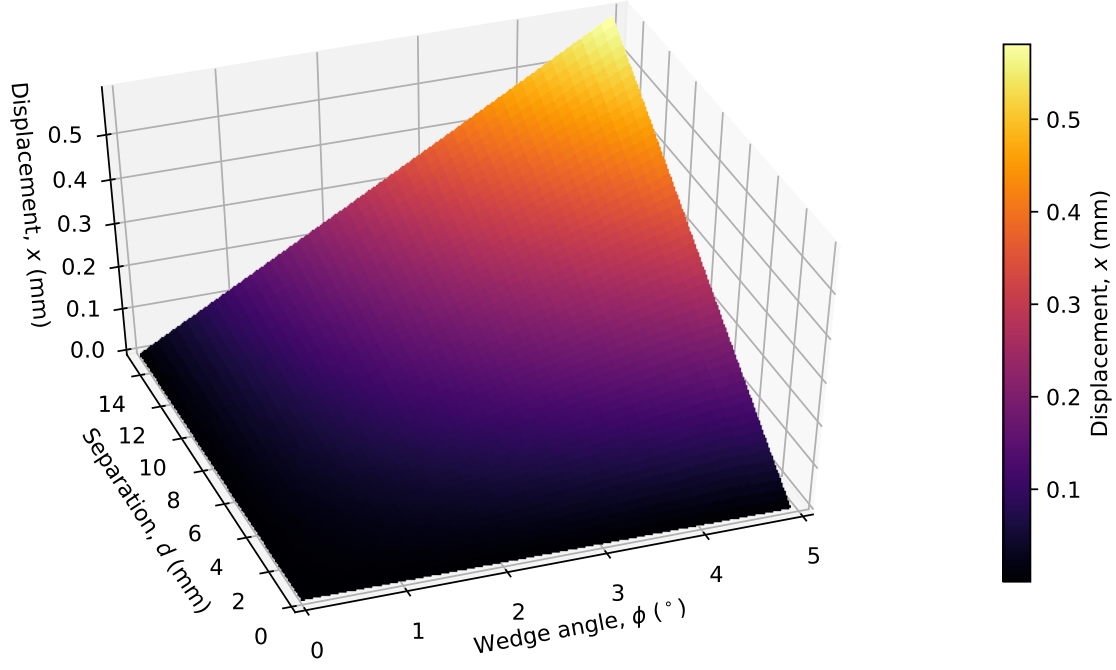

Figure S12: Beam displacement through wedges as a function of wedge angle and separation distance. 


\section{References}

(S1) Brixner, T.; Mančal, T.; Stiopkin, I. V.; Fleming, G. R. Phase-stabilized Twodimensional Electronic Spectroscopy. J. Chem. Phys. 2004, 121, 4221-4236.

(S2) Anna, J. M.; Song, Y.; Dinshaw, R.; Scholes, G. D. Two-dimensional Electronic Spectroscopy for Mapping Molecular Photophysics. Pure Appl. Chem. 2013, 85, 1307.

(S3) Hybl, J. D.; Albrecht Ferro, A.; Jonas, D. M. Two-dimensional Fourier Transform Electronic Spectroscopy. J. Chem. Phys. 2001, 115, 6606-6622.

(S4) Jonas, D. M. Two-dimensional Femtosecond Spectroscopy. Annu. Rev. Phys. Chem. 2003, 54, 425-463.

(S5) Anna, J. M.; Ostroumov, E. E.; Maghlaoui, K.; Barber, J.; Scholes, G. D. Twodimensional Electronic Spectroscopy Reveals Ultrafast Downhill Energy Transfer in Photosystem I Trimers of the Cyanobacterium Thermosynechococcus Elongatus. J. Phys. Chem. Lett. 2012, 3, 3677-3684.

(S6) Born, M.; Wolf, E.; Bhatia, A. B.; Clemmow, P. C.; Gabor, D.; Stokes, A. R.; Taylor, A. M.; Wayman, P. A.; Wilcock, W. L. Principles of Optics: Electromagnetic Theory of Propagation, Interference and Diffraction of Light, 7th ed.; Cambridge University Press, 1999. 